\title{
Increased mast cell numbers in human patellar tendinosis: correlation with symptom duration and vascular hyperplasia
}

\author{
A Scott, ${ }^{1,6} \emptyset$ Lian, ${ }^{2,3}$ R Bahr, ${ }^{3,6}$ D A Hart, ${ }^{4}$ V Duronio, ${ }^{1} \mathrm{~K}$ M Khan ${ }^{1,5,6}$
}

${ }^{1}$ Department of Medicine, University of British Columbia, Vancouver, Canada;

${ }^{2}$ Kristiansund Hospital, Kristiansund, Norway: ${ }^{3}$ Oslo Sport Trauma Research Center, Department of Sports Medicine, Norwegian School of Sport Sciences, Oslo, Norway;

${ }^{4}$ McCaig Center for Joint Injury and Arthritis Research, Faculty of Medicine, University of Calgary; ${ }^{5}$ Department of Family Practice, University of British Columbia, Vancouver, Canada; ${ }^{6}$ Centre for Hip Health, Vancouver Coastal Health Research Institute, Vancouver, Canada

Correspondence to: Dr K Khan, Department of Family Practice, University of British Columbia, 320 - 5950 University Blvd, Vancouver, Canada V6T 1Z3:

kkhan@interchange.ubc.ca

Accepted 8 November 2007

Published Online First

4 March 2008

\begin{abstract}
Objectives: The cellular basis of painful tendon overuse pathology (tendinosis) is poorly understood. It has been suggested that because of the close anatomical associations between mast cells and vessels in connective tissues, mast cells may mediate the development of tendon hypervascularity or oedema.

Objectives: To examine the distribution of mast cells in men and women with patellar tendinopathy.

Design: Case-control study.

Methods: Tendinopathic tissue was collected at open debridement of the patellar tendon and a control tendon from patients undergoing intramedullary nailing of the tibia. The tendon was assessed immunohistochemically by evaluating the distribution of mast cells (AA1), and markers for $T$ lymphocytes (CD3) and macrophages (CD68). The vessel-area fraction was quantified using computer-assisted digital image analysis.
\end{abstract}

Results: The prevalence of mast cells per $\mathrm{mm}^{2}$ (mean 3.3 (SD 3.0)) was greater in tendinosis tissue than in controls (1.1 (1.5); $p=0.036)$. In patients with tendinosis, mast cell density was moderately correlated with the vesselarea fraction $\left(r^{2}=0.49\right)$ and with symptom duration $\left(r^{2}=0.52\right)$.

Conclusion: Mast-cell prevalence in patellar tendinopathy was increased and was predominantly associated with vascular hyperplasia, particularly in patients with longstanding symptoms. Future research should investigate whether mast cells play direct or indirect modulatory roles in the development and progression of human tendinosis.

Repetitive overuse tendon injury (tendinopathy) is a major burden on the healthcare system and a challenge to orthopaedic and sports medicine practitioners. ${ }^{1}$ Clinical symptoms include pain, swelling, and impaired function. ${ }^{12}$ Colour Doppler ultrasound reveals excessive vascular flow in tendinopathy and this finding has been associated with the presence of tendon pain. ${ }^{23}$

Despite the increased vascular flow and vascular hyperplasia that characterise tendinosis, an acute cellular inflammation (neutrophils, macrophages, lymphocytes) is rarely reported in the chronic stages. This lack of typical acute inflammatory cells has led to increasing adoption of the term "tendinosis" as opposed to "tendonitis". ${ }^{4}$ Recently, Schubert et al compared the numbers of CD68+ macrophages, CD3+ T lymphocytes, CD20+ B lymphocytes, and granulocytes in 10 patients with Achilles tendinosis and 10 patients with an acutely ruptured Achilles tendon. In this study, patients with tendinosis displayed increased numbers of macrophages and lymphocytes in association with hypervascular tissue, whereas patients with acutely ruptured tendon displayed greater numbers of granulocytes. ${ }^{5}$ Thus, inflammatory and reparative cell types may play a role in the pathogenesis of tendinosis and the development of vascular hyperplasia in chronic tendon lesions.

Along with macrophages and lymphocytes, mast cell numbers are also known to be increased in a variety of chronic inflammatory or fibrotic disorders. In addition to their well known physiological role in triggering allergic reactions, mast cells release a variety of vasoactive, angiogenic and profibrotic substances that are either stored in granules or synthesised de novo in response to hypoxia, mechanical stimulation, or neurological and inflammatory mediators. ${ }^{6-8}$ Mast-cell products of potential relevance to overuse tendinopathies include histamine, prostaglandins, leucotrienes, growth factors and proteinases. ${ }^{9}{ }^{10}$ Despite this, to our knowledge the distribution of mast cells has not been examined in tendinosis tissue. Therefore, in the current study we examined their distribution in both normal and chronically painful patellar tendons. We hypothesised that because of the anatomical proximity of vessels and mast cells in connective tissues, ${ }^{71}$ mast cells would be more prevalent in tendinosis than in control tendon tissue, and would be correlated with the vessel-area fraction.

\section{METHODS}

The study was approved by the university and hospital research ethics committees, and all patients provided written informed consent.

\section{Participants}

In total, 21 patients (18 men, 3 women; mean age 30.4 years, range 22 to 40 ) and 10 control subjects ( 7 men, 3 women; mean age 28.2 years, range 20 to 42) were included. Patients were athletes who had had at least 3 months of patellar tendon pain and tenderness that was sufficiently severe to prevent them from participating in sports. The mean symptom duration was 22 months (range 5 to 81). MRI confirmed high signal changes corresponding to the area of symptoms (infrapatellar pole). The control group consisted of patients treated with intramedullary nailing for tibial fractures with no current or previous knee pain.

Exclusion criteria were age $<18$ years, previous knee surgery, corticosteroid injections in or around the knee, knee trauma requiring medical attention, and rheumatic or degenerative knee conditions. 


\section{Biopsy procedure}

The surgical exposure was identical in the two groups, with a $5 \mathrm{~cm}$ longitudinal midline or lateral parapatellar incision, splitting of the paratenon and exposure of the patellar tendon. The paratenon was split longitudinally, any pathological paratenon tissue was removed and the tendon was fully exposed. In both groups, the biopsies were taken from the proximal bone-tendon junction. The tendon tissue was excised using a full-thickness, wedge-shaped incision, being widest at the patellar pole and narrowing distally. In the patient group, all abnormal tissue was removed. When abnormal tissue was not evident macroscopically, the excision was based on the location of MRI signal changes. Typically, the surgeon removed a wedge with a proximal base $1 \mathrm{~cm}$ wide and extending to an apex 2$3 \mathrm{~cm}$ distal from the patellar pole. In the control group, the biopsies were taken with a width of at least $5 \mathrm{~mm}$ and a length of at least $20 \mathrm{~mm}$ from the middle portion of the tendon starting at the bone-tendon junction. Immediately after the surgical procedure, biopsies were transferred to Zamboni's fixative where they were stored for 4-24 h, and then washed in $0.1 \mathrm{~mol} / 1$ phosphate-buffered saline (PBS) $\mathrm{pH} 7.2$, with $15 \%$ sucrose $(\mathrm{w} / \mathrm{v})$ and $0.1 \%$ natriumazide. The biopsies were then stored in this solution at $4^{\circ} \mathrm{C}$ for a minimum of $48 \mathrm{~h}$ after which they were embedded in paraffin wax.

\section{Appearance under light microscopy}

Sections of $5 \mu \mathrm{m}$ thickness were cut and stained routinely with $\mathrm{H} \& \mathrm{E}$ (general morphology) and alcian blue (sulphated glycosaminoglycans) and viewed at 100-630 times magnification (Zeiss Axioplan upright microscope; Zeiss, Berne, Switzerland). Areas of adipose or peritendinous tissue were avoided during analysis. Absence of visible tendon pathology with H\&E staining was confirmed in each case in the control group. Tendinosis was defined according to the features of the Bonar histopathological scale. ${ }^{12}$ Briefly, this scale defines tendinosis according to the presence of four features; hypervascularity, collagen disorientation, tenocyte rounding (ie, chondroid metaplasia), and increased glycosaminoglycans. ${ }^{12}$

\section{Labelling of cell types}

The following mouse monoclonal antibodies were used to identify cell types: CD68 (Signet KP1 clone; Cedarlane Laboratories, Hornby, Canada) for macrophages, CD3 (clone F7.2.38; DakoDiagnostics, Glostrup, Denmark) for T lymphocytes, and mast cell tryptase (clone AA1; Dako) for mast cells. ${ }^{13-}$ ${ }_{15}$ Processing for mast cell tryptase was carried out using an autostainer (Dako). The sections were cleared in xylene $(3 \times 15 \mathrm{~min})$, steamed in sodium citrate for $3 \times 5$ minutes, incubated at $37^{\circ}$ with $0.1 \%$ trypsin in $0.1 \%$ calcium chloride $\mathrm{pH} 7.3$ for 5 minutes, quenched for 15 minutes in 3\% hydrogen peroxide, incubated in protein-free blocking solution (Dako) for $15 \mathrm{~min}$, then exposed to $100 \mu \mathrm{l}$ of primary antibody diluted 1:50

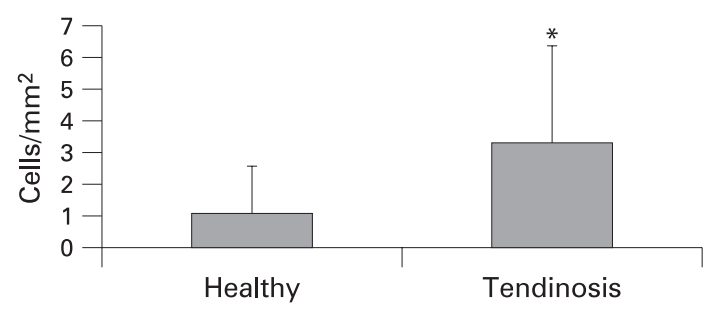

Figure 1 Mast-cell density in normal and tendinosis patellar tendons. ${ }^{*}$ Significance, $p=0.036$. Error bars denote standard deviation. in $0.1 \%$ bovine serum albumin in Tris-buffered saline for 1 hour. A secondary anti-mouse IgG antibody (Dako) was then added, followed by incubation with alkaline phosphatase-anti-alkaline phosphatase (Dako) for 30 minutes. The signal was visualised with new fuchsin (Sigma-Aldritch, Oakville, Canada) as the substrate. Identically fixed and processed tonsil tissue with or without the mast cell tryptase antibody was used as positive or negative control, respectively. CD68 processing was identical to the mast-cell protocol but without the trypsin pre-treatment. For T lymphocytes, the CSA II detection system (Dako) was used, with 3,3'-diaminobenzidine as the chromogen (Vector Laboratories, Burlingame, California, USA). Human tonsil was used as a reference for all cell types. Mast cells, lymphocytes and macrophages were found to be labelled only in the positive tonsil tissue controls.

\section{Image analysis}

Slides were examined with the patient/control identity code masked with black tape. For quantification of mast cell density, the 10 most proximal viewing fields at $\times 40$ magnification were captured in a pre-determined raster pattern, and the number of positive cells per $\mathrm{mm}^{2}$ calculated. Vessel-area fraction was quantified on $\mathrm{H} \& \mathrm{E}$ sections ( 10 most proximal $\times 40$ viewing fields) using image-analysis software (Northern Eclipse; Empix Imaging, Cheektowaga, New York, USA). Vessels were manually outlined onscreen using a mouse, and the area occupied by vessels was expressed as a percentage of the slide area.

\section{Data analysis}

Results are presented as means (SD). Mast-cell density was counted on two separate occasions by a single observer (AS) and averaged (intrarater reliability, $r^{2}=0.75$ ). Mast-cell density in normal and patellar tendinosis tendons was compared using the non-parametric Mann-Whitney $U$ test for independent samples. Correlation between mast cell density and vessel-area fraction, and between mast cell density and symptom duration, were conducted using Pearson's correlation analysis. All statistical tests were carried out using SPSS V.14.0

\section{RESULTS}

\section{Light microscopic appearance}

One control patient had tenocyte rounding in the proximal patellar tendon suggestive of chondroid metaplasia, but no other features of tendinosis pathology. All patients with tendinosis showed all four features of tendinosis pathology as defined by the Bonar histopathological scale: ${ }^{12}$ vascular hyperplasia, collagen disorientation, tenocyte rounding and increased glycosaminoglycan.

\section{Prevalence of mast cells in patient biopsies}

CD3+ lymphocytes and CD68+ macrophages were present too infrequently in either patient or control tendon tissue to be practically quantifiable, despite being readily identified in tonsil tissue. Conversely, there were a greater number of prominent mast cells in patient tendon biopsies. The mast cell density (mean 3.35 (SD 3.5) cells $/ \mathrm{mm}^{2}$ ] was three times greater in tendinosis tissue than in control tissue $\left(1.0\right.$ (1.5) cells $/ \mathrm{mm}^{2}$ ) (fig 1). The mast cells were typically present in a perivascular distribution in areas of increased vessel density within the tendon proper (figs $2-5$ ). In $5 / 22$ cases of tendinosis, and $0 / 10$ controls, mast cells were also occasionally observed within the tendon proper - that is, between longitudinal collagen bundles. 


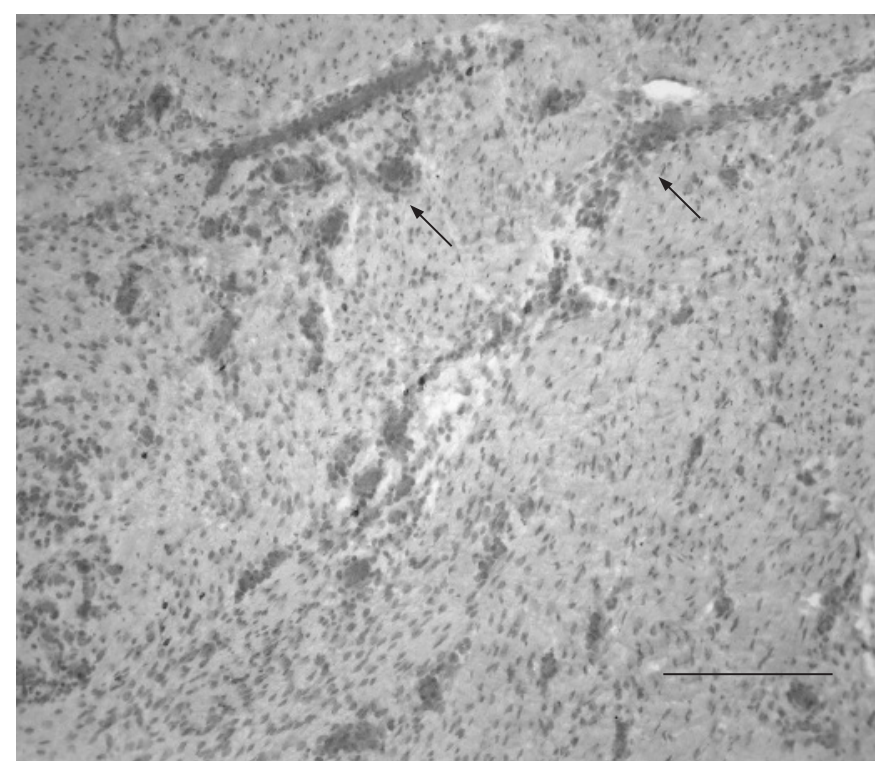

Figure 2 Patellar tendinosis biopsy demonstrating numerous mast cells. Arrows denote examples of microvessels. Note numerous mast cells in association with microvessels. New fuchsin, counter-stained with haematoxylin; original magnification $\times 100$, scale bar $250 \mu \mathrm{m}$.

\section{Correlation of mast cells with tendon vascularity}

Capillaries, arterioles and venules were present in both patient and control biopsies. Patient biopsies consistently showed a greater number of vessels in the posterior, proximal substance of the tendon proper. However, the increase in vascular area $(2.8 \%$ (3.2) in patients vs. $1.2 \%(2.2)$ in controls) was not significant. There was a moderate but significant correlation between mast cells and vessel-area fraction $\left(r^{2}=0.49, p<0.01\right)$.

\section{Correlation of mast cells with symptom duration}

Mast cells were found to be most prominent in biopsies from patients with a longer reported symptom duration. There was a moderate but significant correlation between symptom duration and numbers of mast cells in patients $\left(r^{2}=0.52 ; p<0.05\right)$.

\section{DISCUSSION}

In tendons obtained from symptomatic patellar tendinopathy, the mast cell density was three times higher than in control tendons and was positively correlated with symptom duration. In addition, mast cells were usually found in association with tendon vascularity. In support of this observation, mast cell density was significantly correlated with the vascular area fraction $\left(r^{2}=0.49\right)$. These findings suggest that the accumulation of mast cells in tendinosis may be a feature of the chronic stage of tendon pathology and in associated with vascular hyperplasia.

\section{Novel findings of tendinosis cellularity}

We identified a relatively high number of intratendinous mast cells, the first direct evidence that this cell type is overrepresented in tendinosis tissue compared with control tendon. Classic descriptions of tendinosis tissue obtained from patients undergoing surgery for overuse tendinopathy have not reported prominent mast cells. ${ }^{16-20}$ Increased numbers of mast cells are common in many chronic inflammatory or fibrotic conditions, such as disorders of the joints, gastrointestinal tract, liver and

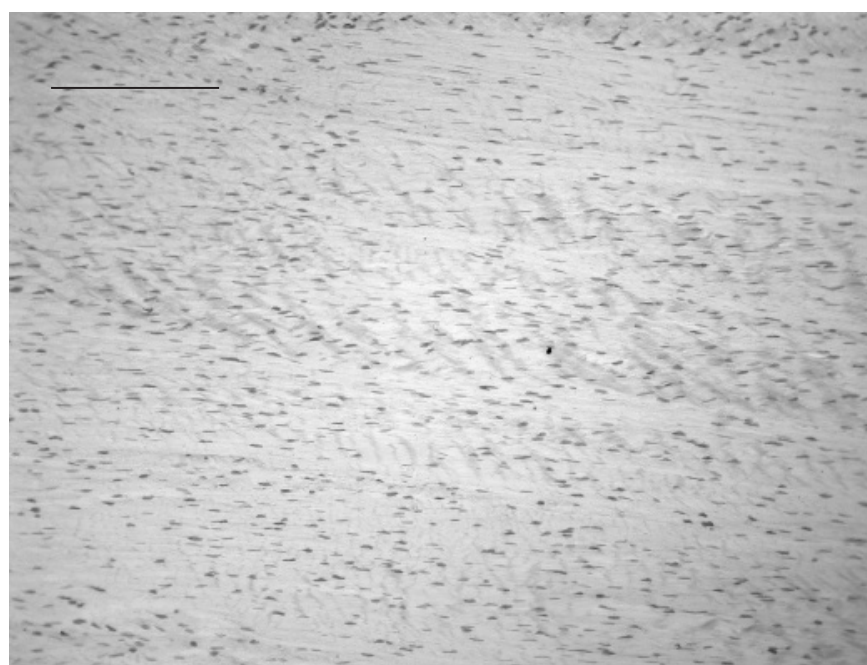

Figure 3 Normal tendon processed identically to tendinosis tendon shown in fig 2. Note lack of microvascular structures in comparison with tendinosis tendon. Original magnification $\times 100$; scale bar $250 \mu \mathrm{m}$.
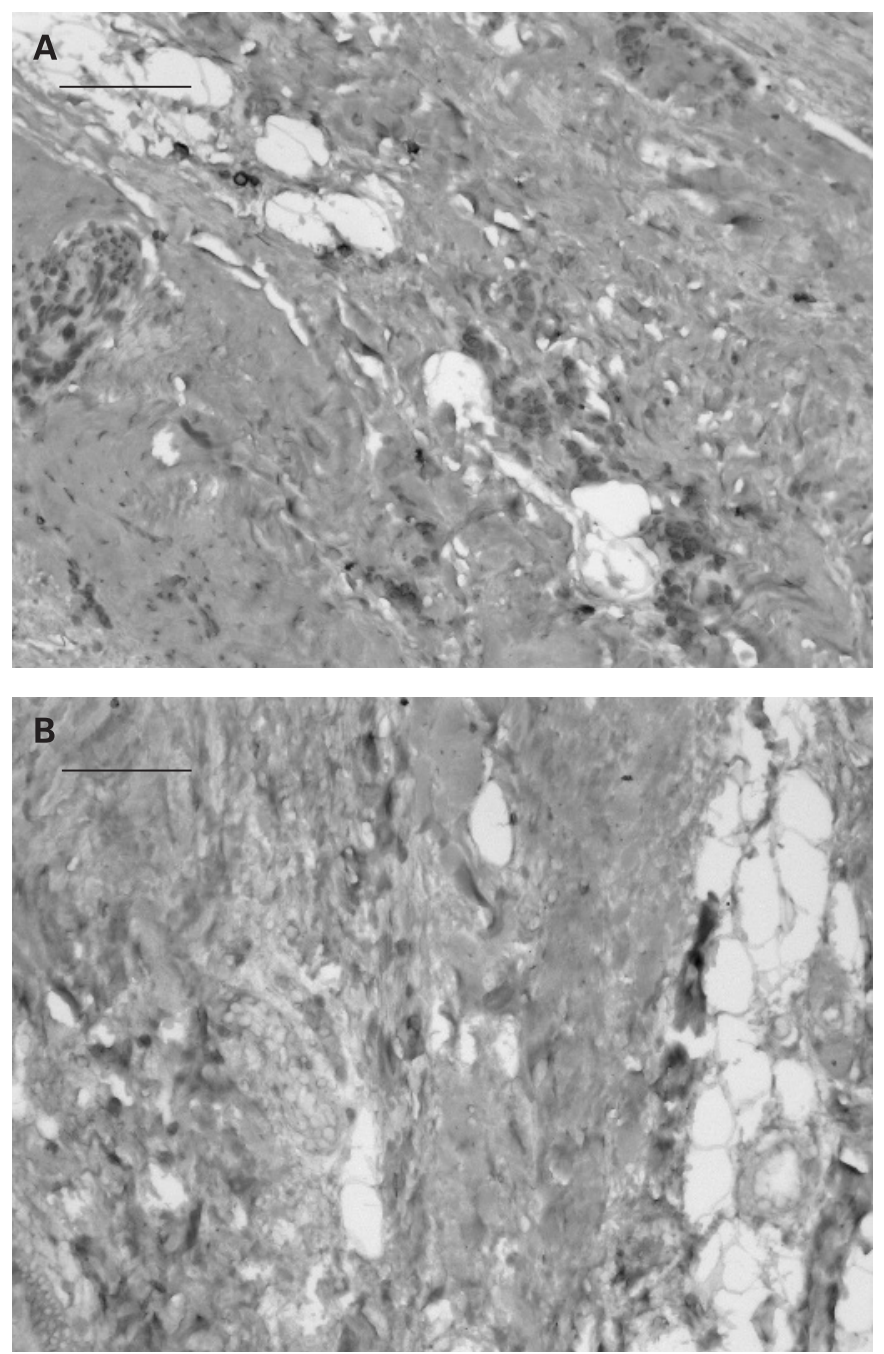

Figure 4 Tendinosis tendon processed $(A)$ with and $(B)$ without antibody to mast cell tryptase. Original magnification $\times 200$; scale bar $100 \mu \mathrm{m}$. 


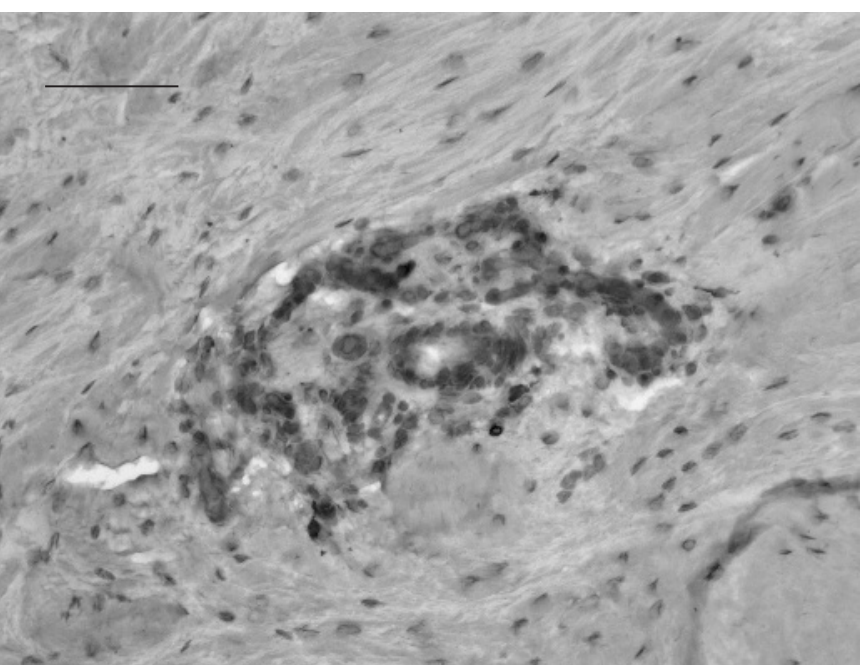

Figure 5 Positively labelled mast cells in association with a microvascular structure in patellar tendinosis biopsy.

respiratory system. Mast cells proliferate at sites of injury and in turn release a range of profibrotic factors including transforming growth factor (TGF) $\beta$ and interleukins 1 and $4 .^{6}$ Mast-cell tryptase can also act directly on fibroblasts by triggering proteinase-activated-receptor-2 (PAR-2), thereby inducing a cyclooxygenase-2-dependent proliferative and fibrotic response in fibroblasts. ${ }^{21}$ Thus, the potential exists for previously undocumented interactions between mast cells and tenocytes.

\section{Potential role of cellular inflammation in tendinosis}

Achilles tendinopathies with symptom duration $<3$ months have been found to have a favourable clinical responses to corticosteroids delivered by iontophoresis, including a reduction in pain and morning stiffness, suggesting the existence of an acute or subacute inflammatory phase that was not captured in the present study. ${ }^{22}$ In support of this clinical observation,

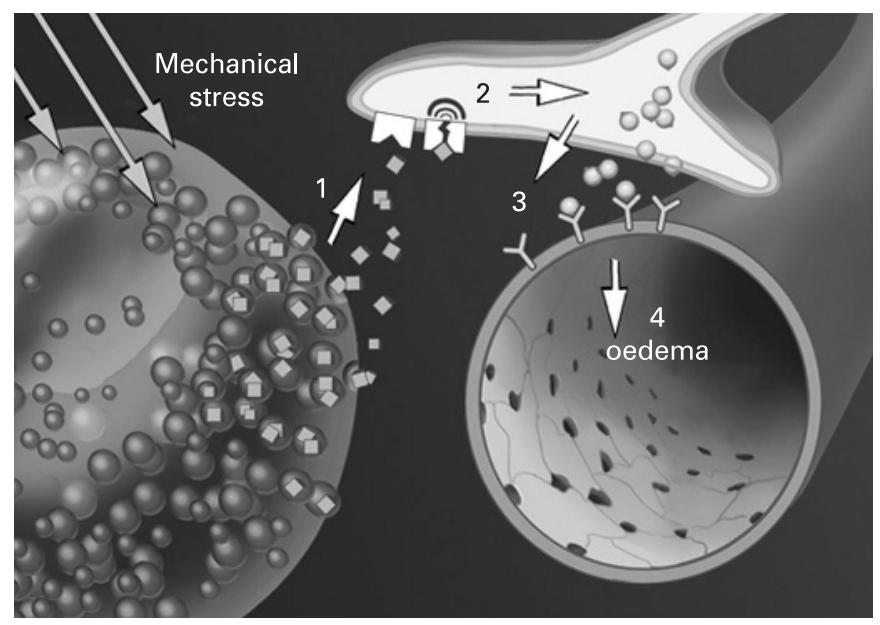

Figure 6 A schematic illustration proposing a potential role of mast cells in the development of tendinosis. In response to mechanical stress (strain, compression, shear), mast cells degranulate and release mast cell tryptase, along with a variety of other vasoactive and angiogenic substances (1). Mast-cell tryptase has the capacity to activate receptors (e.g. proteinase-activated receptor-2) on sensory nerve endings (2). Sensory nerves are capable of releasing SP (3), and this could lead to neurogenic oedema via NK1 receptors (4).

\section{What is already known on this topic}

- Patellar tendinopathy is characterised by increased cellularity, increased vascular flow and angiogenesis.

- In the chronic phase of tendinopathy lesions, acute inflammatory cells are minimally present.

- Mast cells are known to play an important role in soft tissue healing and fibrosis, but their distribution in tendinopathy has not been examined previously.

\section{What this study adds}

- Mast cells are present to a greater degree in patellar tendinopathy, usually in association with microvessels, and particularly in patients with longstanding symptoms.

- Mast cells express tryptase, a potent angiogenic factor, and may therefore play a role in mediating the angiogenic process.

several cellular and molecular features of tendinosis suggest the existence of a chronic inflammatory or fibrotic response including increased expression of TGF $\beta$, increased levels of proteoglycan and collagen III, high intratendinous lactate levels, increased numbers of myofibroblasts, and increased fibroblastic turnover (apoptosis and proliferation). ${ }^{23-30}$ In keeping with these previous studies, the present data show that increased numbers of mast cells, a well-known component of soft tissues in the chronic inflammatory or fibrotic phase of injury, is indeed present in the patellar tendons of patients with symptoms of pain and swelling for $>3$ months, and that the mast cell number may be higher in patients with longer symptom durations.

In contrast to the current study, Schubert et $a l^{5}$ found an increased density of CD68+ macrophages and CD3+ $\mathrm{T}$ lymphocytes in chronically painful Achilles tendons compared with ruptured tendons, which showed an increased density of granulocytes (ie, neutrophils). This suggests that tendinosis may be characterised by an evolving population of invading and locally proliferating cell types, with neutrophils predominant in the early phases, followed by macrophages and lymphocytes, and eventually mast cells and vascular cell types. ${ }^{6}$ Indeed, only a few of the patients in the study by Schubert et al had a symptom duration of $>12$ months, compared with the majority of patients in the current report. This may account for the discrepancy in terms of the number of observable macrophages and lymphocytes between the two studies. A further discrepancy between these two studies is that several of the patients in the Schubert et al study had received corticosteroid injections, which can potentially result in necrosis and focal inflammation. ${ }^{31}$ In contrast, no patient in the current study had received any corticosteroid injection in or around the patellar tendon.

\section{Potential role of neurogenic inflammation in tendinosis}

The mast cells observed in the current study were positive for mast cell tryptase, which is a potent angiogenic factor. ${ }^{11}$ Mast cells are also capable of producing a potent neurotrophin, nerve growth factor, ${ }^{32}$ which is an interesting feature given recent findings that, in addition to angiogenesis, neural sprouting is a prominent feature of tendinosis. ${ }^{5}$ Our cross-sectional study did not allow us to determine whether the prominence of mast cells is causally associated with the abnormal tendon vasculature and 
its accompanying sensory and autonomical innervation, which are strongly implicated in symptomatic tendinopathy. ${ }^{33-38}$ Nonetheless, the findings tempt speculation that mast cells may play proangiogenic and/or neurotrophic roles in tendinopathies. Because the potential exists for multiple paracrine intereactions between mast cells, nerves, endothelial cells, and tenocytes, much work will be required to determine the possible roles of mast cells at different stages in the development and progression of tendinosis. One possible set of interactions is presented in fig 6 .

\section{CONCLUSION}

In summary, we have quantified the density of mast cells in human patellar tendinopathy tissue and found them to be significantly increased in comparison to healthy tendon. Further, we have found that raised mast cell numbers in the patellar tendon are correlated with both the symptom duration and the vessel-area fraction. These observations highlight the need for mechanistic and longitudinal studies to clarify the role of mast cells in tendinopathy.

Acknowledgements: We thank J McDonough for helpful suggestions and A Samrai for excellent technical services, both at the James Hogg iCapture Centre, Vancouver, British Columbia, Canada.

Funding: This work was funded by grants from the Canadian Institutes of Health Research (CIHR) (grant MOP-77551) and the Worker's Compensation Board of British Columbia (grant RS0203-DG-13). The Oslo Sports Trauma Research Center has been established at the Norwegian University of Sport and Physical Education through generous grants from the Royal Norwegian Ministry of Culture, the Norwegian Olympic Committee and Confederation of Sport, Norsk Tipping, and Pfizer. The funding agents played no role in study design or manuscript preparation. AS is a recipient of a $\mathrm{CIHR}$ Post-Doctoral Fellowship. DAH is the Calgary Foundation-Grace Glaum Professor in Arthritis Research and supported by the Institute of Gender and Health of CIHR. VD is a recipient of a Michael Smith Foundation for Health Research Senior Scholar Award. KMK is a recipient of a CIHR New Investigator Award.

Ethics approval: Ethics approval was obtained from the university and hospital ethics committees.

Competing interests: None.

\section{REFERENCES}

1. Hazleman B, Riley G, Speed CA. Soft tissue rheumatology. Oxford: Oxford University Press, 2004.

2. Rees JD, Wilson AM, Wolman RL. Current concepts in the management of tendon disorders. Rheumatology (Oxford) 2006 May;45:508-21.

3. Zanetti M, Metzdorf A, Kundert HP, et al. Achilles tendons: clinical relevance of neovascularization diagnosed with power Doppler US. Radiology 2003 May;227:556-60.

4. Khan KM, Cook JL, Kannus P, et al. Time to abandon the "tendonitis" myth. BMJ 2002 Mar 16;324:626-7.

5. Schubert TE, Weidler C, Lerch $\mathrm{K}$, et al. Achilles tendinosis is associated with sprouting of substance P positive nerve fibres. Ann Rheum Dis 2005 Jul;64:1083-6.

6. Scott A, Khan KM, Roberts CR, et al. What do we mean by the term "inflammation"? A contemporary basic science update for sports medicine. Br J Sports Med 2004:38:372-80.

7. Weber S, Kruger-Krasagakes S, Grabbe J, et al. Mast cells. Int J Dermatol 1995:34:1-10.

8. Kakar S, Khan U, McGrouther DA. Differential cellular response within the rabbit tendon unit following tendon injury. J Hand Surg [Br] 1998;23:627-32.

9. Hart D, Frank CB, Kydd A, et al. Neurogenic, mast cell and gender variables in tendon biology: potential role in chronic tendinopathy. In: Maffulli N, Renstrom P, Leadbetter W, eds. Tendon injuries: basic science and clinical medicine. London: Springer, 2005:40-8.

10. Hart D, Frank C, Bray RC. Inflammatory processes in repetitive motion and overuse syndromes: Potential role of neurogenic mechanisms in tendons and ligaments. In: Fine LJ, ed. Repetitive motion disorders of the upper extremity. Rosemount: American Academy of Orthopaedic Surgeons, 1995:247-62.

11. Blair RJ, Meng H, Marchese MJ, et al. Human mast cells stimulate vascular tube formation. Tryptase is a novel, potent angiogenic factor. J Clin Invest 1997;99:2691-700.
12. Cook JL, Feller JA, Bonar SF, et al. Abnormal tenocyte morphology is more prevalent than collagen disruption in asymptomatic athletes' patellar tendons. J Orthop Res 2004:22:334-8

13. Walls AF, Jones DB, Williams $\mathrm{JH}$, et al. Immunohistochemical identification of mast cells in formaldehyde-fixed tissue using monoclonal antibodies specific for tryptase. $J$ Pathol 1990;162:119-26.

14. Welker $\mathbf{P}$, Grabbe J, Zuberbier T, et al. Mast cell and myeloid marker expression during early in vitro mast cell differentiation from human peripheral blood mononuclear cells. J Invest Dermatol 2000:114:44-50.

15. Walls AF, Bennett AR, McBride HM, et al. Production and characterization of monoclonal antibodies specific for human mast cell tryptase. Clin Exp Allergy 1990;20:581-9.

16. Jarvinen $\mathbf{M}$, Jozsa $L$, Kannus $P$, et al. Histopathological findings in chronic tendon disorders. Scand J Med Sci Sports 1997;7:86-95.

17. Kannus $\mathbf{P}$, Jozsa L. Histopathological changes preceding spontaneous rupture of a tendon. A controlled study of 891 patients. J Bone Joint Surg Am 1991;73:1507-25.

18. Puddu G, Ippolito E, Postacchini F. A classification of Achilles tendon disease. Am J Sports Med 1976;4:145-50.

19. Kvist M, Jozsa L, Jarvinen MJ, et al. Chronic Achilles paratenonitis in athletes: a histological and histochemical study. Pathology 1987:19:1-11.

20. Kraushaar BS, Nirschl RP. Tendinosis of the elbow (tennis elbow). Clinical features and findings of histological, immunohistochemical, and electron microscopy studies. J Bone Joint Surg Am 1999:81:259-78.

21. Frungieri MB, Weidinger $S$, Meineke $V$, et al. Proliferative action of mast-cell tryptase is mediated by PAR2, COX2, prostaglandins, and PPARgamma: Possible relevance to human fibrotic disorders. Proc Natl Acad Sci U S A 200212;99:15072-7.

22. Neeter C, Thomee R, Silbernagel KG, et al. Iontophoresis with or without dexamethazone in the treatment of acute Achilles tendon pain. Scand J Med Sci Sports 2003;13:376-82

23. Fu SC, Wang W, Pau HM, et al. Increased expression of transforming growth factorbeta1 in patellar tendinosis. Clin Orthop 2002:174-83.

24. Maffulli N, Ewen SW, Waterston SW, et al. Tenocytes from ruptured and tendinopathic achilles tendons produce greater quantities of type III collagen than tenocytes from normal achilles tendons. An in vitro model of human tendon healing. Am J Sports Med 2000;28:499-505.

25. Riley GP, Harrall RL, Constant CR, et al. Tendon degeneration and chronic shoulder pain: changes in the collagen composition of the human rotator cuff tendons in rotator cuff tendinitis. Ann Rheum Dis 1994;53:359-66.

26. Alfredson H, Bjur D, Thorsen K, et al. High intratendinous lactate levels in painful chronic Achilles tendinosis. An investigation using microdialysis technique. J Orthop Res 2002:20:934-8.

27. Khan KM, Bonar F, Desmond PM, et al. Patellar tendinosis (jumper's knee): findings at histopathologic examination, US, and MR imaging. Victorian Institute of Sport Tendon Study Group. Radiology 1996;200:821-7.

28. Rolf CG, Fu BS, Pau A, et al. Increased cell proliferation and associated expression of PDGFRbeta causing hypercellularity in patellar tendinosis. Rheumatology (Oxford) 2001;40:256-61.

29. Lian 0, Scott A, Engebretsen $L$, et al. Excessive apoptosis in patellar tendinopathy in athletes. Am Sports Med 2007:35:605-11.

30. Kjaer M. Role of extracellular matrix in adaptation of tendon and skeletal muscle to mechanical loading. Physiol Rev 2004 Apr:84:649-98.

31. Tillander B, Franzen $\mathrm{LE}$, Karlsson $\mathrm{MH}$, et al. Effect of steroid injections on the rotator cuff: an experimental study in rats. J Shoulder Elbow Surg 1999;8:271-4.

32. Marinova T, Philipov S, Aloe L. Nerve growth factor immunoreactivity of mast cells in acute involuted human thymus. Inflammation 2007;30:38-43.

33. Alfredson $\mathbf{H}$, Ohberg $L$, Forsgren $\mathrm{S}$. Is vasculo-neural ingrowth the cause of pain in chronic Achilles tendinosis? An investigation using ultrasonography and colour Doppler, immunohistochemistry, and diagnostic injections. Knee Surg Sports Traumatol Arthrosc 2003:11:334-8.

34. Forsgren S, Danielson P, Alfredson H. Vascular NK-1 receptor occurrence in normal and chronic painful Achilles and patellar tendons: studies on chemically unfixed as well as fixed specimens. Regul Pept 200530:126:173-81.

35. Danielson P, Alfredson H, Forsgren S. Distribution of general (PGP 9.5) and sensory (substance P/CGRP) innervations in the human patellar tendon. Knee Surg Sports Traumatol Arthrosc 2006:14:125-32.

36. Danielson P, Alfredson H, Forsgren S. Immunohistochemical and histochemical findings favoring the occurrence of autocrine/paracrine as well as nerve-related cholinergic effects in chronic painful patellar tendon tendinosis. Microsc Res Tech 2006;69:808-19.

37. Danielson $\mathbf{P}$, Alfredson $\mathrm{H}$, Forsgren $\mathrm{S}$. Studies on the importance of sympathetic innervation, adrenergic receptors, and a possible local catecholamine production in the development of patellar tendinopathy (tendinosis) in man. Microsc Res Tech 2007;70:310-24.

38. Alfredson H, Forsgren S, Thorsen K, et al. Glutamate NMDAR1 receptors localised to nerves in human Achilles tendons. Implications for treatment? Knee Surg Sports Traumatol Arthrosc 2001:9:123-6. 